\title{
Vehicle state estimation based on Kalman filters
}

\author{
M. Bersani, M. Vignati, S. Mentasti, S. Arrigoni, F. Cheli \\ Politecnico di Milano, Dept. of Mechanical Engineering \\ via G. La Masa, 1 - 20156 Milano Italy \\ Email: mattia.bersani@polimi.it \\ michele.vignati@polimi.it \\ simone.mentasti@polimi.it \\ stefano.arrigoni@polimi.it \\ federico.cheli@polimi.it
}

\begin{abstract}
Vehicle state estimation represents a prerequisite for ADAS (Advanced Driver-Assistant Systems) and, more in general, for autonomous driving. In particular, algorithms designed for path or trajectory planning require the continuous knowledge of some data such as the lateral velocity and heading angle of the vehicle, together with its lateral position with respect to the road boundaries. Vehicle state estimation can be assessed by means of extended and unscented Kalman filters (EKF and UKF, respectively), that have been well treated in the literature. Referring to an experimental case study, the presented work deals with the design and the real time implementation of two different adaptive Kalman filters for vehicle sideslip and positioning estimation. Accuracy have been assessed by means of an automotive optical sensor.

Index Terms-State estimation, Autonomous driving, EKF, UKF
\end{abstract}

\section{INTRODUCTION}

Active safety systems become more and more spread and sophisticated leading gradually to the commercialisation of completely autonomous vehicles. The development of autonomous driving proceeds rapidly even if the legislation is still weak and unclear, moreover, people do not seen favourably the possibility that the car drives itself [1]. This side of public opinion is strengthened by some resounding facts as the one happened in Tempe, Arizona, where a pedestrian was struck and killed by a car operating in autonomous driving mode. These are obviously symptoms of a technology that needs a further improvement to become completely reliable and safe. Nevertheless, these technologies are crucial, because as reported by [1] and [2], about 37000 people die every years only in the United States.

Autonomous driving control loop bases its effectiveness on the deep knowledge of the current vehicle motion. Accordingly, information like velocities in centre of gravity ( $\operatorname{cog}$ ), heading angle and positioning with respect to road centreline and boundaries are mandatory. State estimation on vehicles becomes fundamental since Electronic Stability

Published in the context of the project TEINVEIN: TEcnologie INnovative per i VEicoli Intelligenti, CUP (Codice Unico Progetto - Unique Project Code): E96D17000110009 - Call "Accordi per la Ricerca e IInnovazione", cofunded by POR FESR 2014-2020 (Programma Operativo Regionale, Fondo Europeo di Sviluppo Regionale Regional Operational Programme, European Regional Development Fund).
Program (ESP) was born, given that information like yaw rate are crucial for the consequent feedback control on vehicle handling [2].

Nowadays, different methodologies are available to estimate the state of a vehicle, especially for the side-slip angle and the lateral velocity, because they are difficult to be measured. In this paper, we focus on the real time estimation of the lateral and longitudinal velocities, the heading angle of the vehicle and its absolute position, aiming to ensure the autonomous driving control loop.

Lateral velocity can be simply derived by means of a pure kinematic single-track model as illustrated in [3]: the author considers an ideal case in which pure rolling contact is considered, thus its formulation requires only the information about longitudinal velocity and the steering angle. This approximated model can be considered valid up to $5 \mathrm{~m} / \mathrm{s}$. In [4], authors present the comparison between three different methods: the first is a transfer function of two states, lateral velocity and yaw rate, while the available measurements are the steering angle and the yaw rate. The second one is a state-space approach of the system. Both of them rely on a dynamic model of the vehicle. About the third and last one, it considers a pure kinematic single-track model that estimates the lateral and longitudinal velocities instead of the yaw rate. All these methods suffer when the road-bank angle is not flat, so they must be enhanced by a properly road-bank estimation algorithm. Moreover the first two are also dependent on vehicle parameters, as the cornering stiffness, that can vary according to the actual conditions of the vehicle and the road surface.

Imsland in [5] proposes a modular non linear observer to estimate the longitudinal and lateral velocities measuring accelerations, yaw rate, steering angle and wheels speed. It is shown how the model is easily tunable and it could be an alternative to the Kalman filter, because it reduces the computational effort by avoiding the resolution of the Riccati equations. However the strong dependency from the friction coefficient, that must be imposed, limits its robustness. The work presented in [6] describes two different methods for the lateral velocity estimation: a physic one based on the handling system modelling and a kinematic one. 
In particular, the first one includes a correction that makes the model insensitive to parameters variations; nevertheless, both of them are not able to correctly estimate the lateral speed in the non-linear handling region. This issue is faced by means of kinematic relationships. Selmanaj [7], starting from the second approach, introduces an heuristic term to correct the estimation at null values of yaw rate, afterwards, the longitudinal speed measured on the wheels is corrected considering some kinematic effects and the longitudinal wheel slip. Both approaches are based on a 6DOF Inertial Measurement Unit (IMU), wheel speed sensors and steering angle measurement. Another proposal based on a kinematic vehicle model is presented in [8], that relies on the acceleration equations with respect to a rotating frame. It solves the estimation problem by applying an algebraic technique for the numerical differentiation and diagnosis.

These authors did not correct their estimations by means of Global Positioning System (GPS), that represents a real economic advantage allowing a cheaper setup. Moreover, the GPS can be a powerful tool to improve the estimation of the vehicle position with respect to the road boundaries. Some researchers, as Ryu and Gerdes [9], highlight the possibility of integrating the measurements provided by IMUs with the GPS. They rely on a single track kinematic model and a Kalman filter to account for both the signals. Two well distinct filters are illustrated: one for the yaw angle and another one for the lateral and longitudinal velocities. Proceeding step by step they are refined considering first the road-bank angle and then the correction of the bias. Moreover, the implemented Kalman filter handles the different sampling frequencies of IMU and GPS.

Similarly, authors in [10] propose to integrate the GPS with an Inertial Navigation System (INS) sensors to obtain more reliable estimation and faster update. Two different setups are described: the former one includes only a GPS receiver, while the latter one relies on two GPS receivers placed along the transversal axis of the car to measure both yaw and roll motion of the vehicle. The second solution exploits the best results because it accounts for the roll motion. Both systems implement the INS sensors as input and the GPS signals as measurements. A Kalman filter is then applied to the system to estimate the state of the vehicle. Two GPS receivers are used also in [11]. However, authors suggest to locate the receivers along the longitudinal axis of the vehicle. The aim is to estimate the longitudinal and lateral velocity and the yaw angle without using the position of the GPS but only their velocities. Also in this case a Kalman filter is implemented to integrate the measurements from the GPS and the input from the IMU, basing on a kinematic single track model.

Starting from the last proposal, this paper presents the implementation of an adaptive extended and unscented Kalman filters (EKF and UKF respectively) to allow estimating the velocities of the vehicle in local reference system and concurrently the absolute position. Both the filters are based on a single track kinematic model to avoid the

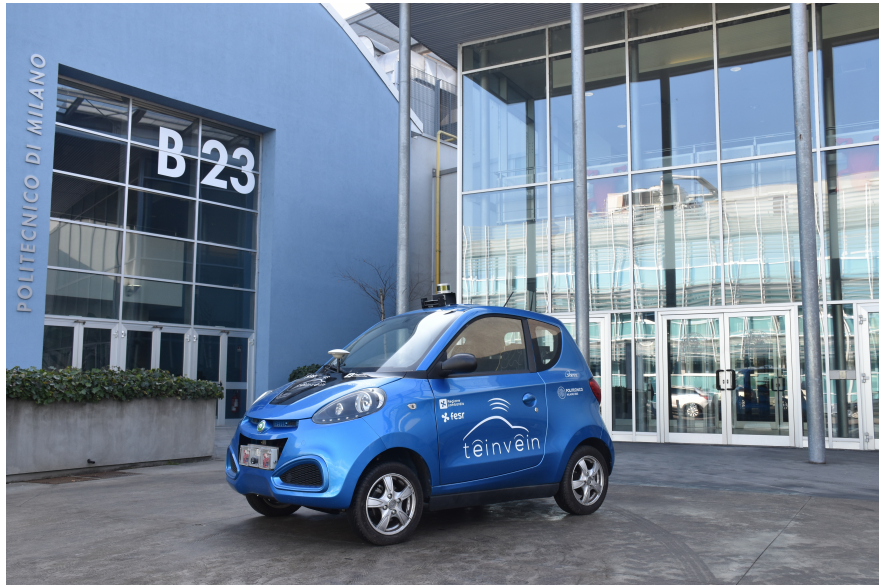

Fig. 1. The experimental vehicle

dependency from the vehicle dynamic parameters, aiming at guarantee ease of use and portability.

In general, many works on state estimation presented in literature are based on dynamic modelling of vehicles [12], [13], [14]. Indeed, they allow improving the prediction of the process and concurrently avoiding some issues as the drift due to integration of the sensors bias [13]. On the other hand, dynamic modelling requires accurate vehicle parameters estimation, such as mass, moment of inertia and tyre cornering stiffness to avoid errors [12]. To overcome this, hybrid modelling is presented in [13], in which sensor-based estimation is adopted to compensate uncertainties due to dynamic parameters and friction coefficients. Moreover the filter is adaptive, since it accounts for time dependency of the covariance error matrices to improve reliability and robustness. However, this level of complexity is not suitable for real time application, as stated by authors. Similar results are obtained in [14], where a kinematic model is integrated with the information provided by a dynamic model (i.e. vehicle model based filter, VMF) and by the GPS receiver. To ensure the real time state estimation within an autonomous driving control loop in urban environment, it can be stated that a kinematic vehicle model is a good compromise between reliability and accuracy.

The paper is organized as follows: in section II the experimental vehicle and the measurement system are presented, section III provides some remarks of the implementation process of EKF and UKF for real time applications, while section IV presents the experimental results. To conclude, the last section provides a summary of the paper with future developments.

\section{THE EXPERIMENTAL SETUP}

The instrumented vehicle is shown in Fig.1: a front wheel drive full-electric quadricycle, powered by a $6 \mathrm{~kW}$ AC motor. The energy source consists on a HV battery (150 Ah @ $72 \mathrm{~V}$ ) which can be charged only during a plug-in phase 
TABLE I

VEHICLE PARAMETERS

\begin{tabular}{cc|l} 
Mass & $\mathrm{kg}$ & 668 \\
Wheelbase & $\mathrm{mm}$ & 1765 \\
Track & $\mathrm{mm}$ & 1450 \\
Range & $\mathrm{km}$ & 120
\end{tabular}

(i.e. BEV - battery electric vehicle). The braking system is composed by four disc-brakes without any ABS or ESP control unit. Table I reports some important measurements. Authors recommend works presented in [15] and [16] for a wider discussion on measurements and modifications carried on the presented vehicle.

Measurements that have been used for the state estimation discussed in this work are provided by:

- two GPS receivers located along the longitudinal axis of the vehicle;

- an automotive IMU placed in the estimated centre of gravity $(\operatorname{cog})$;

- two tone wheels installed within the disk brakes on the rear shaft;

- an incremental optical encoder connected to the DC motor of the EPS (Electronic Power Steering) system of the vehicle.

The GPS receivers accounts for the RTK (real time kinematic) correction if associated with a ground station. Nevertheless, without any correction they guarantee a position error in the order of $0.8 \mathrm{~m}$, while the error associated with the velocity provided in the ENU (East-North-Up) reference system is in the order of $0.03 \mathrm{~m} / \mathrm{s}$. The update frequency is about $10 \mathrm{~Hz}$ by means of ETH communication. The automotive IMU is a 5-axis sensor that provides the linear accelerations along the three principal axis of the vehicle and concurrently the rotational speed around the $\mathrm{x}$ and $\mathrm{z}$ axis (i.e. the roll and yaw rate respectively). The sensor communicates on CAN-bus with an update frequency of $100 \mathrm{~Hz}$. The measurements of the steering angle and of the longitudinal velocity of the vehicle are provided respectively by the incremental optical encoder on the EPS system and the tone wheels at the rear axle. These kinematic measurements are provided to the estimation unit through serial communication (at $20 \mathrm{~Hz}$ ) by a hard real time system which is responsible for the vehicle low-level actuation.

\section{NONLINEAR STATE ESTIMATION}

The extended and unscented KFs allow dealing with a nonlinear model of the process dynamics. Aiming at guarantee the real time implementation of the filter, we refer to the respective discrete-time implementations. The state vector (1) includes the longitudinal and lateral velocities in the vehicle cog (according to the kinematic single track model [3]), together with the overall heading angle. Furthermore, information about positioning are given.

$$
\mathbf{x}=\left[\begin{array}{lllll}
V_{x} & V_{y} & \psi & x_{g} & y_{g}
\end{array}\right]^{T}
$$

In both the filters, information provided by the IMU are considered as input for the kinematic model of the vehicle. Equations of motions are listed in (2), where $a_{x}, a_{y}$ and $\omega$ accounts for the measured longitudinal and lateral accelerations and yaw rate.

$$
\left\{\begin{array}{l}
\dot{V}_{x}=V_{y} \dot{\psi}+a_{x} \\
\dot{V}_{y}=-V_{x} \dot{\psi}+a_{y} \\
\dot{\psi}=\omega \\
\dot{x}=V_{x} \cos \psi-V_{y} \sin \psi \\
\dot{y}=V_{x} \sin \psi+V_{y} \cos \psi
\end{array}\right.
$$

In the previous equations, velocities are referred to the local reference system centred in the vehicle $\operatorname{cog}$, while positioning is provided in the absolute reference system. To conclude, inputs given by the IMU are provided without the sensor bias that can be measured when the vehicle is standstill during the initialization phase.

The measurement equations integrate the velocities and positions provided by the GPS receivers together with the state variables in (1). In particular, kinematic measurements of the longitudinal and lateral velocities are required to accounts for accuracy of the satellite signal. As anticipated in the previous section, they are provided by the tone wheels at the rear axle and by the encoder on the EPS system. Although they do not take into account the variations of rolling radius, longitudinal slip, and concurrently the nonlinear geometry of the rackand-pinion transmission, kinematic relationships are reliable on vehicles in the speed-range of interest [3].

Accordingly, since the vehicle is front wheel-steering only, the lateral speed in the cog can be computed as follow:

$$
V_{y}=l_{r} / l_{w b} \cdot \tan (\delta) \cdot V_{x}
$$

where $l_{r}$ accounts for the distances between $\operatorname{cog}$ and the rear axle, while $l_{w b}$ is the wheelbase, and $\delta$ represents the steering angle at the wheels.

To conclude, measurements equations are reported in (4), in which $V_{f e}, V_{f n}, V_{r e}, V_{r n}$ represents the velocities in ENU coordinates provided by the two GPS receivers.

$$
\left\{\begin{array}{l}
V_{f e}=V_{x} \cos \psi-\left(V_{y}+l_{f} \dot{\psi}\right) \sin \psi \\
V_{f n}=V_{x} \sin \psi+\left(V_{y}+l_{f} \dot{\psi}\right) \cos \psi \\
V_{r e}=V_{x} \cos \psi-\left(V_{y}-l_{r} \dot{\psi}\right) \sin \psi \\
V_{r n}=V_{x} \sin \psi+\left(V_{y}-l_{r} \dot{\psi}\right) \cos \psi \\
V_{x_{w}}=V_{x} \\
V_{y_{w}}=V_{y} \\
x_{g}=x \\
y_{g}=y
\end{array}\right.
$$


The discrete time implementation of EKF and UKF are based on the nonlinear systems reported in (5).

$$
\begin{aligned}
x_{k+1} & =f\left(x_{k}, u_{k}, t_{k}\right)+w_{k} \\
y_{k} & =h\left(x_{k}, t_{k}\right)+v_{k} \\
w_{k} & \sim\left(0, Q_{k}\right) \\
v_{k} & \sim\left(0, R_{k}\right)
\end{aligned}
$$

In particular, the previous equations account for additive process disturbance $w_{k}$ and measurement noise $v_{k}$. They have been modelled as zero mean distributed with covariance matrices $Q_{k}$ and $R_{k}$ respectively.

The $Q_{k}$ matrix is considered as constant and diagonal, whose elements have been calculated taking into account the square value of the expected maximum variation for each state variable (6). On the other hand, $R_{k}$ is positive definite and it changes during time according to different covariance calculations on a moving window of length equal to $1 \mathrm{~s}$.

$$
Q_{k_{i i}}=\frac{1}{\left(\mathbf{x}_{i_{\max }}-\mathbf{x}_{i_{\text {min }}}\right)^{2}}
$$

For what concerns the EKF, a first order Taylor expansion is performed on the state and measurement equations. The Jacobian matrix of the kinematic vehicle model is evaluated as:

$$
\begin{aligned}
& F_{k-1}=\left[\begin{array}{ccc}
\frac{\partial \dot{V}_{x}}{\partial V_{x}} & \ldots & \frac{\partial \dot{V}_{x}}{\partial y} \\
\vdots & \ddots & \vdots \\
\frac{\partial \dot{y}}{\partial V_{x}} & \cdots & \frac{\partial \dot{y}}{\partial y}
\end{array}\right]^{\hat{x}_{k-1}^{+}}= \\
& {\left[\begin{array}{ccccc}
0 & \dot{\psi} & 0 & 0 & 0 \\
-\dot{\psi} & 0 & 0 & 0 & 0 \\
0 & 0 & 0 & 0 & 0 \\
\cos (\psi) & -\sin (\psi) & -V_{x} \sin (\psi)-V_{y} \cos (\psi) & 0 & 0 \\
\sin (\psi) & \cos (\psi) & V_{x} \cos (\psi)-V_{y} \sin (\psi) & 0 & 0
\end{array}\right]^{\hat{x}_{k-1}}}
\end{aligned}
$$

while the Jacobian matrix of the measurement equations is represented as:

$$
C_{k}=\left[\begin{array}{ccc}
\frac{\partial \dot{V}_{f e}}{\partial V_{x}} & \ldots & \frac{\partial \dot{V}_{f e}}{\partial y} \\
\vdots & \ddots & \vdots \\
\frac{\partial \dot{y}_{g}}{\partial V_{x}} & \cdots & \frac{\partial \dot{y}_{g}}{\partial y}
\end{array}\right]^{\hat{x}_{k}^{-}}=
$$

$\left[\begin{array}{ccccc}\cos (\psi) & -\sin (\psi) & -V_{x} \sin (\psi)-\left(V_{y}+l_{f} \dot{\psi}\right) \cos (\psi) & 0 & 0 \\ \sin (\psi) & \cos (\psi) & V_{x} \cos (\psi)-\left(V_{y}+l_{f} \dot{\psi}\right) \sin (\psi) & 0 & 0 \\ \cos (\psi) & -\sin (\psi) & -V_{x} \sin (\psi)-\left(V_{y}-l_{f} \dot{\psi}\right) \cos (\psi) & 0 & 0 \\ \sin (\psi) & \cos (\psi) & V_{x} \cos (\psi)-\left(V_{y}-l_{f} \dot{\psi}\right) \sin (\psi) & 0 & 0 \\ 1 & 0 & 0 & 0 & 0 \\ 0 & 1 & 0 & 0 & 0 \\ 0 & 0 & 0 & 1 & 0 \\ 0 & 0 & 0 & 0 & 1\end{array}\right]$

Finally, after the update of the state vector and the respective measurement error, EKF provides the state estimation by means of the following equations.

$$
\begin{gathered}
K_{k}=P_{k}^{-} C_{k}^{T}\left(C_{k} P_{k}^{-} C_{k}^{T}+R_{k}\right)^{-1} \\
\hat{x}_{k}^{+}=\hat{x}_{k}^{-}+K_{k}\left[y_{k}-h_{k}\left(\hat{x}_{k}^{-}\right)\right] \\
P_{k}^{+}=\left(1-K_{k} C_{k}\right) P_{k}^{-}
\end{gathered}
$$

Thus, Kalman gain in (9) depends also on the $R_{k}$ measurements noise covariance matrix, that is considered as time dependent. Concurrently, $y_{k}$ in equation (10) represents the measurements provided at time $k$, while $h_{k}$ accounts for the propagation of the state vector $\hat{x}_{k}^{-}$to the measurement equations.

As explained in [17], the unscented Kalman filter (UKF) is based on the intuition that is easier to approximate a probability distribution rather than an arbitrary nonlinear function or transformation. Although the EKF is the most widely applied state estimation algorithm for nonlinear systems, it may bring to undesirable errors if the nonlinearities in the system are severe. This happens because EKF works on the principle that a linearized transformation of mean and covariance is approximately equal to the true nonlinear transformation. On the other hand, UKF accounts for the ease of perform a nonlinear transformation on a single point rather than an entire distribution. Furthermore, the unscented transformation allows defining a sample of points whose distribution well approximates the true one. As explained in [18], [17], the approximated mean of a state vector matches the true mean correctly up to the third order, whereas linearization only matches the true mean up to the first order. For what concerns the state vector covariance, the unscented transformation approximates the true one up to the third order, that is the same result obtained in EKF. Nevertheless, it can be expected that the magnitude of the error can be lower in UKF, since the sample of points which belong to the transformation includes contributions of a higher order.

A comparison between EKF and UKF is provided in [19], where both the filters are applied to a double-track vehicle dynamic model. The numerical and experimental results confirm that the two filters demonstrate similar performances when the sample time is lower than $5 \mathrm{~ms}$. However, the estimate provided by UKF at higher timings results more accurate.

After the UKF has been initialized, to propagate the state estimate and covariance from one measurement to the next, the unscented transformation is applied. The sigma-points (i.e. the sample of points that are chosen for their capability in representing the mean and covariance of the real probability density function) are evaluated according to the following 
equations:

$$
\begin{aligned}
\hat{x}_{k-1}^{(i)} & =\hat{x}_{k-1}^{(+)}+\tilde{x}^{(i)} \quad i \in[1, \ldots, 2 n] \\
\tilde{x}^{(i)} & =\left(\sqrt{n P_{k-1}^{+}}\right)_{i}^{T} \quad i \in[1, \ldots, n] \\
\tilde{x}^{(n+i)} & =-\left(\sqrt{n P_{k-1}^{+}}\right)_{i}^{T} \quad i \in[1, \ldots, n]
\end{aligned}
$$

where $n$ is the number of sigma points, that usually can double the length of the state vector. Then, the nonlinear equations of the vehicle model and the measurement equations transform the sigma points to obtain the update of the estimations. In UKF, the cross covariance matrix between state vector and measurements updates is taken into account while evaluating the Kalman gain matrix (13).

$$
\begin{gathered}
K_{k}=P_{x y} P_{y}^{-1} \\
\hat{x}_{k}^{+}=\hat{x}_{k}^{-}+K_{k}\left[y_{k}-\hat{y}_{k}\right] \\
P_{k}^{+}=P_{k}^{-}-K_{k} P_{y} K_{k}^{T}
\end{gathered}
$$

To accomplish real time estimation, it is required to consider the different sampling frequencies of the sensors involved. In particular, GPS receivers publish the respective measurements at $10 \mathrm{~Hz}$, while kinematic quantities are given at $20 \mathrm{~Hz}$ and IMU provides accelerations and rotational rates at $100 \mathrm{~Hz}$.

Since the closed loop frequency of a trajectory planner can be considered equal to $20 \mathrm{~Hz}$ (as indicated in [20]), state estimation should be provided at least at the same frequency. For this reason, it has been developed a switching filter triggered by the receiving of the serial data timed by the hard real time actuation system. In this way, the update phase accounts for the GPS signal only once every couple of sends. In the remaining samples, positions provided by GPS receivers are integrated in time by means of kinematic relationships.

Both the Kalman filters have been modelled in a Simulink environment that subscribes on a ROS network, which allows receiving measurements from ETH, serial and CAN-bus concurrently.

\section{EXPERIMENTAL RESULTS}

The capability of the described filters in estimating the velocities in the vehicle cog together with the respective heading angle in real time has been assessed by means of experimental measurements provided by an automotive sensor. In particular, the acquisition system is composed by a noncontact optical sensor which measures velocities and heading. As anticipated, the covariance matrix $Q_{k}$ strictly depends on the process, while $R_{k}$ is time dependent and accounts for the variation of the measurements noise covariance. Fig.2, represents the results of the estimation process in terms of longitudinal velocity, lateral velocity and heading angle of the vehicle. In can be observed that kinematic relationships are well suited whilst driving in an urban environment scenarios. About heading angle, in the previous section no equations
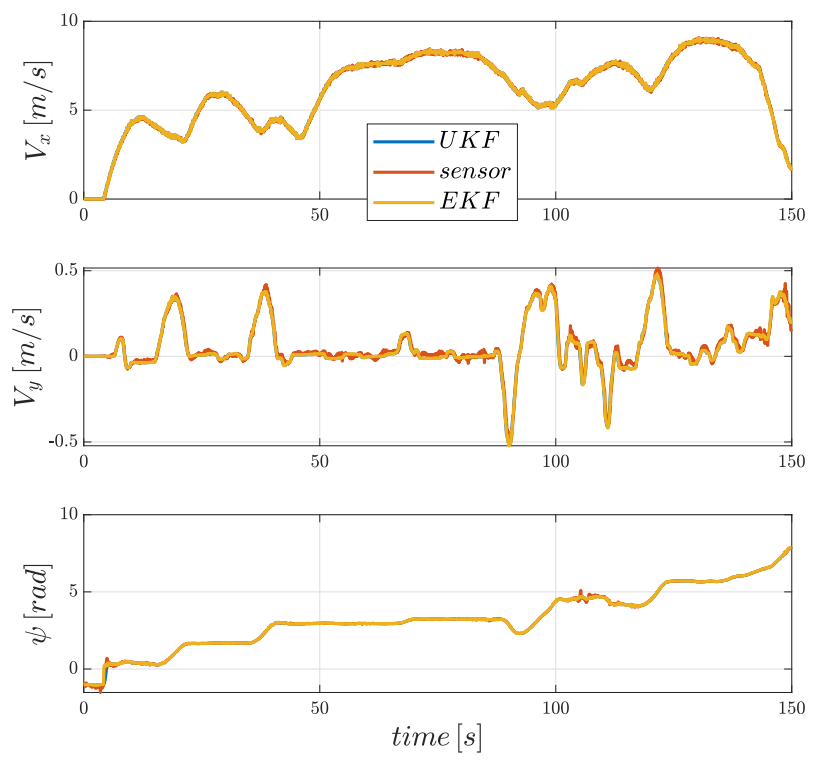

Fig. 2. Vehicle state estimation - validation

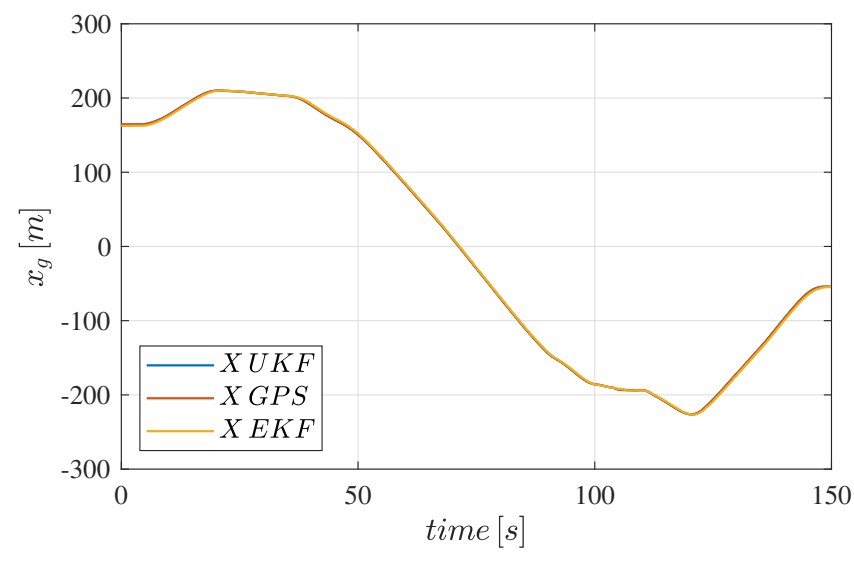

Fig. 3. Vehicle positioning - validation

have been presented that considers the heading angle as a measure. This happened because the high error covariance of the signals provided by the GPS receivers, that have not been corrected with RTK during this work to improve robustness. Nevertheless, the heading angle estimated by EKF and UKF is compared with the measured one in Fig.2, that is provided by the optical sensor (and filtered in post-process with a secondorder low-pass filter without phase shift). To conclude the validation phase, Fig. 3 compares the absolute position of the vehicle on the $\mathrm{x}$ axis of a global reference system during time with the values estimated by the filters.

\section{Conclusions}

The presented paper describes the implementation of an adaptive EKF and UKF for the real time estimation of velocities, heading angle and positioning of an experimental 
vehicle. Both the filters are based on a kinematic single track vehicle model, in which inputs are given by an IMU located in the vehicle cog, while two GPS receivers, two tone wheels at rear axle and an encoder connected to the steering shaft provide the measurements for estimation. To assess real time effectiveness in autonomous driving, the estimate must be provided at $20 \mathrm{~Hz}$, that doubles the sampling frequency of the GPS receivers. This oversampling is handled by triggering two similar filters at the target frequency: the first one accounts for the GPS measurements, while in the second one positions are integrated basing on kinematic relationships.

Both the filters have been implemented in Simulink on a Ubuntu-driven laptop, from which a preliminary time analysis shown that they require less than $0.025 \mathrm{~s}$ to provide the estimate. Accuracy have been assessed by means of experimental measurements provided by an automotive optical sensor.

This preliminary study validates the capability of EKF and UKF in estimating the state of a vehicle for real time applications. In the next steps, accuracy will be improved and more significant time analysis will be carried on both the filters. To conclude, a significant benchmark for the comparison of EKF and UKF performances will be defined.

\section{REFERENCES}

[1] J.Barkenbus, "Self-driving cars: how soon is soon enough?" Issues in science and technology, vol.34, no.4, 2018.

[2] A. Van Zanten and R. Bosch Gmbh, "Evolution of electronic control systems for improving vehicle dynamic behaviour," in Proceedings of the International Symposium on Advanced Vehicle Control (AVEC), 2002.

[3] R. Rajamani, Vehicle Dynamics and Control. Springer, 2012.

[4] A. Y. Ungoren and H. Peng, "A study on lateral speed estimation," International Journal of Vehicle Autonomous Systems, Vol. 2, 2004.

[5] L. Imsland, T. A. Johansen, T. I. Fossen, J. C. Kalkkuhl, and A. Suissa, "Vehicle velocity estimation using modular nonlinear observers," in Proceedings of the 44th IEEE Conference on Decision and Control, Dec 2005, pp. 6728-6733.

[6] J. Farrelly and P. Wellstead, "Estimation of vehicle lateral velocity," in Proceeding of the 1996 IEEE International Conference on Control Applications IEEE International Conference on Control Applications held together with IEEE International Symposium on Intelligent Control, Sep. 1996, pp. 552-557.

[7] D. Selmanaj, M. Como, G. Panzani, and S. Savaresi, "Robust Vehicle Sideslip Estimation Based on Kinematic Considerations," in IFAC PapersOnline, Volume 50 Issue 1, 2017.

[8] J. Villagra, B. d'Andrea-Novel, M. Fliess, and H. Mounier, "Estimation of longitudinal and lateral vehicle velocities: An algebraic approach," in 2008 American Control Conference, June 2008.

[9] J. Ryu and J. Christian Gerdes, "Integrating inertial sensors with global positioning system (gps) for vehicle dynamics control," Journal of Dynamic Systems Measurement and Control-transactions of The Asme - J DYN SYST MEAS CONTR, vol. 126, 062004.

[10] D. M. Bevly, J. Ryu, and J. C. Gerdes, "Integrating ins sensors with gps measurements for continuous estimation of vehicle sideslip, roll, and tire cornering stiffness," IEEE Transactions on Intelligent Transportation Systems, vol. 7, no. 4, pp. 483-493, Dec 2006.

[11] J. Yoon and H. Peng, "A cost-effective sideslip estimation method using velocity measurements from two gps receivers," IEEE Transactions on Vehicular Technology, vol. 63, no. 6, pp. 2589-2599, July 2014.

[12] T. Wenzel, K. Burnham, M. Blundell, and R. Williams, "Dual extended kalman filter for vehicle state and parameter estimation," Vehicle System Dynamics - VEH SYST DYN, vol. 44, pp. 153-171, 022006.

[13] J. Chen, J. Song, L. Li, G. Jia, X. Ran, and C. Yang, "Ukf-based adaptive variable structure observer for vehicle sideslip with dynamic correction," IET Control Theory Applications, vol. 10, no. 14, pp. 1641-1652, 2016.
[14] X. Li, C. Chan, and Y. Wang, "A reliable fusion methodology for simultaneous estimation of vehicle sideslip and yaw angles," IEEE Transactions on Vehicular Technology, vol. 65, no. 6, pp. 4440-4458, June 2016.

[15] M. Vignati, D. Tarsitano, and F. Cheli, "On how to transform a commercial electric quadricycle into a full autonomously actuated vehicle," in International Symposium on Advanced Vehicle Control, 07 2018, pp. $1-5$.

[16] M. Vignati, D. Tarsitano, M. Bersani, and F. Cheli, "Autonomous steer actuation for an urban quadricycle," in 2018 International Conference of Electrical and Electronic Technologies for Automotive, 07 2018, pp. $1-5$.

[17] S. J. Julier and J. K. Uhlmann, "Unscented filtering and nonlinear estimation," in Proceedings of the IEEE, vol. 92, no. 3, March 2004.

[18] D.Simon, Optimal State Estimation: Kalman, H Infinity, and Nonlinear Approaches. Wiley, 2006.

[19] S. Antonov, A. Fehn, and A. Kugi, "Unscented kalman filter for vehicle state estimation," Vehicle System Dynamics, vol. 49, pp. 1497-1520, 09 2011.

[20] S. Arrigoni, E. Trabalzini, M. Bersani, F. Braghin, and F. Cheli, "Nonlinear mpc motion planner for autonomous vehicles based on accelerated particle swarm optimization algorithm," in 2019 International Conference of Electrical and Electronic Technologies for Automotive, 072019. 